\title{
Enhanced NMR Relaxation of Tomonaga-Luttinger Liquids and the Magnitude of the Carbon Hyperfine Coupling in Single-Wall Carbon Nanotubes
}

\author{
A. Kiss, ${ }^{1,2}$ A. Pályi, ${ }^{3}$ Y. Ihara,${ }^{4}$ P. Wzietek, ${ }^{4}$ P. Simon, ${ }^{4}$ H. Alloul,${ }^{4}$ V. Zólyomi,,${ }^{2,5}$ \\ J. Koltai, ${ }^{6}$ J. Kürti, ${ }^{6}$ B. Dóra, ${ }^{1}$ and F. Simon ${ }^{1,7, *}$ \\ ${ }^{1}$ Budapest University of Technology and Economics, Institute of Physics and Condensed Matter Research Group \\ of the Hungarian Academy of Sciences, H-1521 Budapest, Hungary \\ ${ }^{2}$ Research Institute for Solid State Physics and Optics of the Hungarian Academy of Sciences, P.O. B. 49, H-1525, Budapest, Hungary \\ ${ }^{3}$ Department of Materials Physics, Eötvös University, Pázmány Péter sétány 1/A, H-1117 Budapest, Hungary \\ ${ }^{4}$ Laboratoire de Physique des Solides, Université Paris-Sud 11, UMR 8502, 91405 Orsay, France \\ ${ }^{5}$ Department of Physics, Lancaster University, Lancaster, LA1 4YB, United Kingdom \\ ${ }^{6}$ Department of Biological Physics, Eötvös University, Pázmány Péter sétány 1/A, H-1117 Budapest, Hungary \\ ${ }^{7}$ Universität Wien, Fakultät für Physik, Strudlhofgasse 4, 1090 Wien, Austria
}

(Received 8 June 2011; published 27 October 2011)

\begin{abstract}
Recent transport measurements [Churchill et al. Nature Phys. 5, 321 (2009)] found a surprisingly large, $2-3$ orders of magnitude larger than usual ${ }^{13} \mathrm{C}$ hyperfine coupling (HFC) in ${ }^{13} \mathrm{C}$ enriched single-wall carbon nanotubes. We formulate the theory of the nuclear relaxation time in the framework of the Tomonaga-Luttinger liquid theory to enable the determination of the HFC from recent data by Ihara et al. [Europhys. Lett. 90, 17004 (2010)]. Though we find that $1 / T_{1}$ is orders of magnitude enhanced with respect to a Fermi-liquid behavior, the HFC has its usual, small value. Then, we reexamine the theoretical description used to extract the HFC from transport experiments and show that similar features could be obtained with HFC-independent system parameters.
\end{abstract}

Albeit small, the electron-nuclear hyperfine coupling (HFC) is the dominant mechanism in physical phenomena which are key to, e.g., nuclear quantum computing [1], magnetic resonance spectroscopy [2], and it plays a fundamental role in spintronics [3]. The HFC is due to the magnetic interaction between the nucleus and electrons, with a number of different mechanisms such as the Fermi contact, spin-dipole, core-polarization, orbital, and transferred HFC [4].

It is generally accepted that the HFC does not change more than an order of magnitude for different environments of an atom [5]. Typical values for the ${ }^{13} \mathrm{C}$ HFC are $1-5 \times 10^{-7} \mathrm{eV}[6-8]$ with a largest known value of $1.8 \times$ $10^{-6} \mathrm{eV}$ in an organic free radical [9].

It therefore came as a surprise that transport experiments [10] on a double quantum dot formed of ${ }^{13} \mathrm{C}$ enriched single-wall carbon nanotubes (SWCNTs) found a HFC, $A=1-2 \times 10^{-4} \mathrm{eV}$, which is $2-3$ orders of magnitude larger than measured for $\mathrm{C}_{60}$ [7] or calculated for graphene [8], which are similar carbonaceous nanostructures. In Ref. [10], a theory developed for GaAs quantum dots [11] was used to analyze the data, which has some shortcomings. First, the HFC in GaAs is $2-3$ orders of magnitude stronger than the usual value in carbon. Second, both Ga and As have a nearly isotropic HFC [12] whereas the anisotropic HFC usually dominates for carbon [7]. Third, SWCNTs possess an extra degree of freedom, the so-called valley degeneracy, which may lead to distinct behavior of the QD transport properties [13]. Fourth, the particular one dimensionality of SWCNTs may affect the analysis. Clearly, settling the issue calls for an analysis which yields the HFC directly from magnetic resonance spectroscopy.

NMR measurement of the ${ }^{13} \mathrm{C}$ spin-lattice relaxation time, $T_{1}$, in SWCNTs can give directly the HFC and such results were reported in Refs. [14-17]. However, the analysis requires care since the Fermi-liquid (FL) theory for $T_{1}$ does not apply in the SWCNTs as the low energy excitations in metallic SWCNTs are described by the Tomonaga-Luttinger liquid (TLL) framework [18-21]. The TLL is an exotic correlated state [22,23] and yet SWCNTs offer its best realization. So far theory focused on the unusual temperature, $T$, dependence of $T_{1}$ but its magnitude has not been explained [24].

Here, we develop the theory of the NMR spin-lattice relaxation rate for a TLL including anisotropic HFC. We show that $T_{1}$ found in the NMR reports is not compatible with a Fermi-liquid description even if earlier reports argued for this state $[14,15]$. For a TLL, the NMR relaxation rate is orders of magnitude enhanced compared to a FL with the same density of states (DOS) and HFC. The HFC is determined from the $T_{1}$ data and it is found to be as small as $3.6 \times 10^{-7} \mathrm{eV}$ in clear contrast to the transport data in Ref. [10]. Based on this result, we reexamine the features in the transport data which were used to infer the HFC strength in Ref. [10] and show that they might as well be interpreted with a theory which does not depend on the HFC.

We first identify the theoretical model for the NMR $T_{1}$ data analysis. The $T$ dependence for the FL and TLL 
descriptions is markedly different: $\left(T_{1} T\right)^{-1}$ is a constant for a FL (the so-called Korringa law [2]) but it shows a power law $T$ dependence for a TLL [24]. Unfortunately, the behavior of $\left(T_{1} T\right)^{-1}$ found experimentally is conflicting: earlier studies found a $T$ independent $\left(T_{1} T\right)^{-1}$ for metallic SWCNTs [14,15], whereas latter ones clearly showed a power-law behavior $[16,17]$. Given that recent studies agree $[20,21,25]$ about the validity of the TLL description, the discrepancy between the NMR results is probably due to the inferior quality of the earlier samples. This means that the $T_{1}$ data have to be described within the TLL framework.

We now turn to the quantitative description of the NMR $T_{1}$ data. First, we show that a FL description cannot explain the magnitude of the experimental $T_{1}$. We consider a hyperfine Hamiltonian of the most general anisotropic form:

$$
H_{\mathrm{HFC}}=\mathbf{S} \overline{\mathbf{A}} \mathbf{I},
$$

where $\mathbf{S}$ and $\mathbf{I}$ are the electron- and nuclear-spin operators, $\overline{\mathbf{A}}$ is a $3 \times 3$ tensor with diagonal elements of which the traceless ones are due to the spin-dipole interaction as $A_{\text {dip }}(x, y): A_{\text {dip }}(z)=-A_{\text {dip }}: 2 A_{\text {dip }}$ and the scalar term is $A_{\text {iso }}$. The angular average of the NMR relaxation rate for a FL is [2]

$$
\left\langle\left(T_{1} T\right)_{\mathrm{FL}}^{-1}\right\rangle_{\Omega}=A_{\mathrm{eff}}^{2} \frac{\pi k_{B}}{\hbar} \rho^{2} \beta^{-1},
$$

where $\langle\ldots\rangle_{\Omega}$ denotes angular averaging, $A_{\text {eff }}^{2}=A_{\text {iso }}^{2}+$ $2 A_{\text {dip }}^{2}$ is an effective HFC, $\rho$ is the DOS in units of states/(eV $\cdot$ atom $\cdot$ spin) at the Fermi energy, $E_{F}$ [26].

The factor $\beta^{-1}$ is one for a Fermi gas and it accounts for correlation effects but it remains below 20 even for systems such as, e.g., $\mathrm{YBa}_{2} \mathrm{Cu}_{3} \mathrm{O}_{7}$ which displays strong antiferromagnetic fluctuations.

At $T=300 \mathrm{~K}$, values of $T_{1}=9$ [14] and $5 \mathrm{sec}$ [17] were reported. It was argued in Ref. [14] that this $T_{1}$ can be explained using $\rho=0.022$ states $/(\mathrm{eV} \cdot$ atom $\cdot$ spin $)$ and $A_{\text {dip }}=8.2 \times 10^{-7} \mathrm{eV}$. However, both of these numbers are overestimates; i.e., this DOS is about 3 times larger than the results of first principles calculations and the HFC is also about a factor 2 too large [27]. We summarize the literature values of $A_{\text {eff }}$, the DOS, and $T_{1}$ in Table I. DOS for representative SWCNTs with diameters around $1.4 \mathrm{~nm}$ are also given therein, which are calculated with first principles using the density functional theory (DFT) (details are provided in [29]).

Clearly, the experimental and the $T_{1}$ values calculated in the FL picture differ by orders of magnitude, even if we consider the combination which gives the shortest calculated $T_{1}$ of $330 \mathrm{sec}$. Alternatively, one should invoke an unphysically large $\beta^{-1} \approx 30$ to explain the data.

In the following, we discuss the NMR relaxation in the TLL picture. The determination of $T_{1}$ follows from an expansion of the transition rate in the HFC using Fermi's golden rule, and the resulting general expression is
TABLE I. Hyperfine coupling constants (in $10^{-7} \mathrm{eV}$ units) and density of states (in units of $10^{-3}$ states $/(\mathrm{eV} \cdot$ atom . spin)), and experimental and calculated $T_{1}$ (in seconds) at $T=$ $300 \mathrm{~K}$.

\begin{tabular}{lcccc}
\hline \hline Quantity & & $A_{\text {eff }}$ & $\rho$ & $T_{1}$ \\
\hline HFC & C $60_{60}$ [7] & 5.32 & & \\
& graphene [8] & 3.53 & & \\
DOS for SWCNT & tight-binding [28] & & 7 & \\
& DFT & & 7 & \\
$T_{1}$ Exp. on SWCNT & Ref. [14] & & & 9 \\
& Ref. [17] & & & 5 \\
$T_{1}$ Calc. for a Fermi liquid & Refs. [7,29] & & & 330 \\
\hline \hline
\end{tabular}

$$
\left\langle\left(T_{1} T\right)^{-1}\right\rangle_{\Omega}=A_{\text {eff }}^{2} \frac{2 k_{B}}{\hbar} \sum_{q} \operatorname{Im} \frac{\chi_{+-}\left(q, \omega_{0}\right)}{\hbar^{2} \omega_{0}},
$$

where $\omega_{0}$ is the nuclear Larmor frequency and $\chi_{+-}\left(q, \omega_{0}\right)=\int d t \int d x \exp (i(\omega t+q x) \times$

$\left\langle\left[S^{+}(x, t), S^{-}(0,0)\right]\right\rangle$ is the transversal dynamic susceptibility. In a TLL, the separated charge and spin excitations are characterized by the TLL parameters, $K_{c}$ and $K_{s}$ [30]. Assuming spin rotational invariance (i.e., $K_{s}=1$ ), $\chi_{+-}\left(q, \omega_{0}\right)$ is isotropic and the angular averaging in Eq. (3) only involves the anisotropic HFC. Based on Ref. [24], we obtain the NMR relaxation rate with HFC anisotropy for a TLL:

$$
\left\langle\left(T_{1} T\right)_{\mathrm{TLL}}^{-1}\right\rangle_{\Omega}=A_{\text {eff }}^{2} \frac{1}{\hbar k_{B}}\left(\frac{2 \alpha a \pi k_{B}}{\hbar v_{F}}\right)^{K} T^{K-2} C(K),
$$

where $a$ is the lattice constant, $v_{F}$ is the Fermi velocity, $K=K_{c}+1 / K_{s}$ and $C(K)=\sin (\pi K) \Gamma(1-$ $K) \Gamma^{2}(K / 2) / 2$ is a dimensionless constant with values between 5 and 1.5 for $1<K<2$. $K=1.34$ was measured for SWCNTs in Ref. [17]. The relation of the $K$ exponent to the charge, $K_{c}$, and spin, $K_{s}$, Luttinger parameters is given in the supplementary information [29], in particular, considering the relevance of one- and two-band Luttinger models. We omit the angular averaging symbol in the following.

Here we introduced the dimensionless short distance cutoff, $\alpha$, regularizing the theory in the continuum limit, at the expense of retaining the dimensionful lattice constant, $a$. Later on, $\alpha$ is estimated by comparing the results of the FL with the TLL state at the noninteracting point $K=2$.

We rewrite Eq. (4) for a metallic SWCNT with $(n, m)$ chiral index and a linear energy dispersion of $\epsilon(k)=\hbar v_{F} k$. For SWCNTs, it is known that the DOS depends on the diameter and thus on the $(n, m)$ indices and it is $\rho(n, m)=$ $\frac{a_{0}}{\pi \hbar v_{F}} \Xi(n, m)$ in units of states $/(\mathrm{eV} \cdot$ atom $\cdot$ spin) [28] with $\Xi(n, m)=\frac{\sqrt{3}}{2 \sqrt{\left(n^{2}+m^{2}+n m\right)}}$. The relaxation rate in the TLL picture reads: 


$$
\left(T_{1} T\right)_{\mathrm{TLL}}^{-1}=A_{\mathrm{eff}}^{2} \frac{\pi k_{B}}{\hbar}\left(\frac{2 \alpha \pi^{2}}{\Xi(n, m)}\right)^{K} \frac{C(K)}{\pi}\left[\rho k_{B} T\right]^{K-2} \rho^{2}
$$

and expressing it in terms of the FL relaxation rate in Eq. (2):

$$
\frac{\left(T_{1} T\right)_{\mathrm{TLL}}^{-1}}{\left(T_{1} T\right)_{\mathrm{FL}}^{-1}}=\left(\frac{2 \alpha \pi^{2}}{\Xi(n, m)}\right)^{K} \frac{C(K)}{\pi}\left[\rho k_{B} T\right]^{K-2} .
$$

The constant $\alpha$ depends on $(n, m)$ and is obtained by setting $K=2$ when the right-hand side of Eq. (6) is one. Then $C(2)=\pi / 2$ and for a $(10,10)$ SWCNT it gives $\alpha=$ $\sqrt{2} / 40 \pi^{2} \approx 3.6 \times 10^{-3}$.

We observe a speeding up of the NMR relaxation rate when going from the FL to the TLL. The low $\rho=6.9 \times$ $10^{-3}$ states $/(\mathrm{eV} \cdot$ atom $\cdot \operatorname{spin})$ gives $\left[\rho k_{B} T\right]^{K-2} \approx 300$ at room temperature for the SWCNTs with $K=1.34$. This can be qualitatively understood as if the electrons were partially localized in the TLL state as in the Heisenberg model [30] which contributes to a fluctuating, $T^{-1}$ like $T$ dependence of the NMR relaxation rate. The numerical prefactor, $\left(\frac{2 \alpha \pi^{2}}{\Xi(n, m)}\right) \frac{C(K)}{\pi}$ is $\sim 0.8$ for $K=1.34$ and stays near unity.

The present theory of the NMR relaxation rate allows us to compare it quantitatively with the experiment. In Fig. 1., we show the experimental NMR relaxation rate in SWCNTs at $3 \mathrm{~T}$ from Ref. [17]. A fit with the above model in Eq. (5) gave $A_{\text {eff }}=3.6 \times 10^{-7} \mathrm{eV}$ when we fixed $K=$ 1.34 as in Ref. [17] and the DOS to the first principles based value of $\rho=0.007$ states $/(\mathrm{eV} \cdot$ atom $\cdot$ spin $)$. This value of the effective hyperfine coupling is in good agreement with the literature values for other carbonaceous materials shown in Table I.

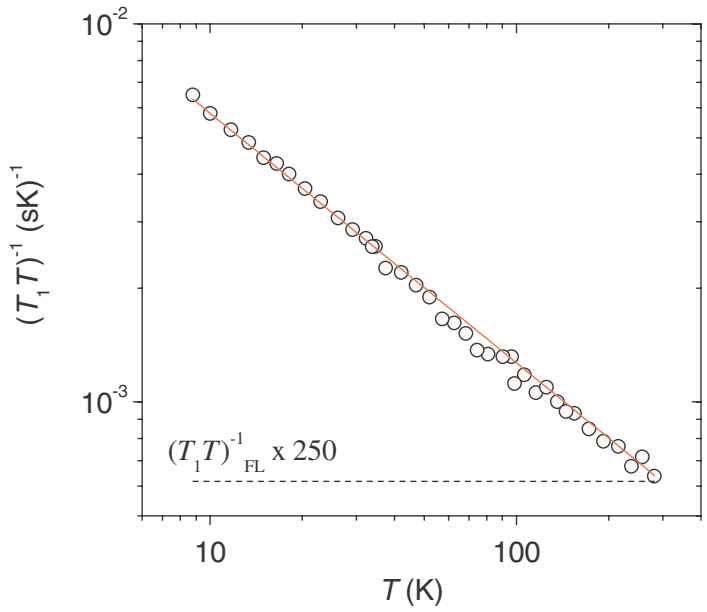

FIG. 1 (color online). The temperature normalized NMR relaxation rate in SWCNTs at $3 \mathrm{~T}$ (symbols) from Ref. [17] fitted with the TLL model (solid line). The relaxation rate calculated in a Fermi-liquid picture, $\left(T_{1} T\right)_{\mathrm{FL}}^{-1}$ (dashed line), is shown multiplied by a factor of 250 .
The NMR line position also provides a measure of the HFC. For a TLL with anisotropic HFC, the Knight shift, $S_{i}$ $(i=x, y, z)$, reads:

$$
S_{i}=A_{i i} \frac{\gamma_{e}}{2 \gamma_{\mathrm{C}}} \rho K_{s},
$$

where the $A_{i i}$ 's are the diagonal elements of the HFC tensor. Equation (7) retains the FL result for SWCNTs since therein $K_{s}=1$. For the powder SWCNT samples, the first moment of the NMR line is $A_{\text {iso }}$. The NMR data in Ref. [31] give an upper limit of $20 \mathrm{ppm}$ for the isotropic Knight shift which leads to $A_{\text {iso }}<2.2 \times 10^{-6} \mathrm{eV}$ with the $\rho$ values in Table I. We note that microscopic calculations also find a small value of the HFC in SWCNTs; $A_{\text {iso }}=$ $2.2 \times 10^{-7} \mathrm{eV}$ was found in Ref. [32] and $A \approx 10^{-6} \mathrm{eV}$ was reported in Ref. [33]. While the TLL theory applies to describe the low energy behavior such as it is relevant for the NMR experiments, a sizeable energy level spacing in quantum dots can significantly affect the result [34].

We now turn to discuss the transport experiments on a double quantum dot (DQD) formed of ${ }^{13} \mathrm{C}$ enriched SWCNTs [10], which found the HFC as large as $A=$ $1-2 \times 10^{-4} \mathrm{eV}$ in marked contrast to the above value of $A_{\text {eff }} \simeq 10^{-6} \mathrm{eV}$ obtained from the NMR data. The possibility to obtain the HFC from transport measurements on DQDs arises from the fact that the weak nuclear fields enable otherwise forbidden tunneling currents between the two dots, an effect called lifting of spin-blockade (LSB) $[35,36]$. This effect is now well established in GaAs quantum dots with a theory provided by Jouravlev and Nazarov (JN) [11]. In Ref. [10], the LSB was observed for the ${ }^{13} \mathrm{C}$ enriched SWCNTs and a quantitative analysis was performed with a direct application of the JN theory. We discuss herein the essentials of the theory and present a potential alternative interpretation of the experimental results.

The JN theory starts by pointing out that the electron feels a "frozen" nuclear field in each dot since the nuclear relaxation times are much longer than any relevant time scale for electrons. This condition remains valid for SWCNTs. The nuclear field in each dots is treated as a classical variable with values of $\mathbf{B}_{N}^{L}$ and $\mathbf{B}_{N}^{R}$ (for the left and right dots) with a Gaussian distribution [37]: $\left\langle\left(\mathbf{B}_{N}^{L(R)}\right)^{2}\right\rangle_{G}=B_{N}^{2}$. Here, $g \mu_{B} B_{N}=A I / \sqrt{N}$ with $g \approx 2$ being the electron $g$ factor, $\mu_{B}$ is the Bohr magneton, and $N$ is the number of ${ }^{13} \mathrm{C}$ nuclei in each dot $[11,38]$.

Figure 2(a) shows the geometry and the energy structure of a DQD. The energy spectrum of the DQD consists of a ground state with single occupancy, followed by a doubly occupied two-site state (either singlet or triplet, separated by an energy $E$ ) and an on-site singlet state $(S)$ (separated by $E+\Delta$ ), and finally an even higher lying on-site triplet state. Assuming an electron with spin up trapped in the right dot, only an electron with down spin can hop from the left side to this dot forming the on-site singlet state because 
a)
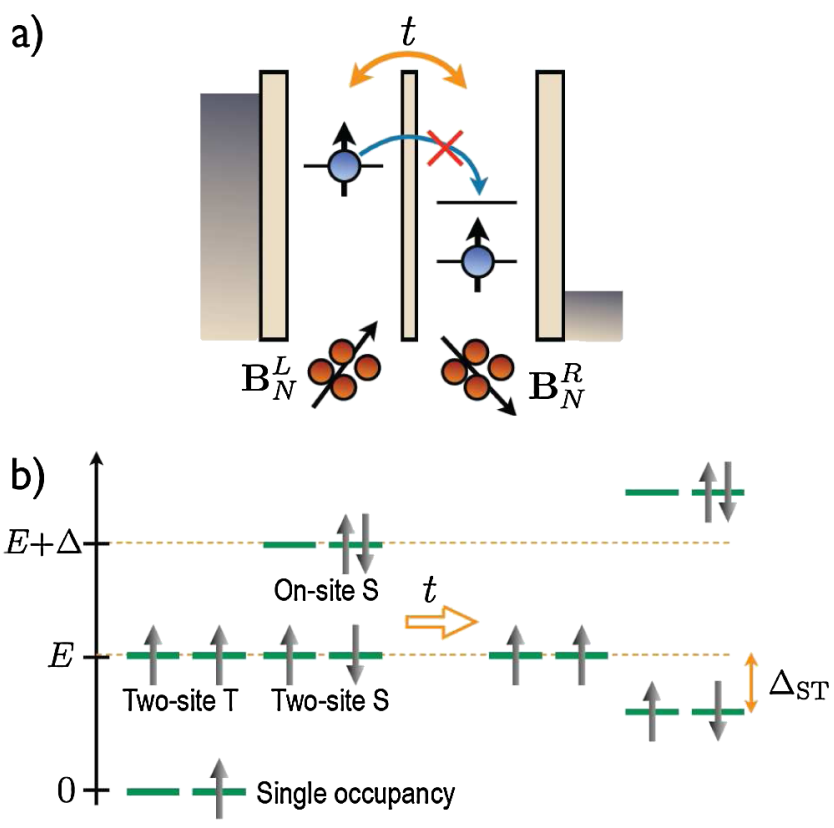

FIG. 2 (color online). (a) Schematics of a DQD; the contact leads (grey areas) and the internal hyperfine fields in the left and right dots are indicated. (b) Energy levels for the DQD. Note that the tunneling parameter couples the on-site $S$ and two-site $S$ states and results in the singlet-triplet splitting.

spin-flip transitions are forbidden and the high energy onsite triplet state is not realized. Thus, for the two-dot system, two-electron spin singlet and triplet states can be identified. For the earlier, the transport is possible, whereas it is blocked for the latter. These states are separated by a singlet-triplet splitting, $\Delta_{\mathrm{ST}}[39]$.

Without external magnetic and nuclear fields, $\Delta_{\mathrm{ST}}$ is determined by $\Delta$ and the interdot tunneling matrix element $t$ : assuming large detuning $\Delta \gg t$, one finds $\Delta_{\mathrm{ST}} \approx t^{2} / \Delta$. Lifting of spin-blockade occurs when the difference of the nuclear fields between the two quantum dots, $\mathbf{B}_{N}^{L}-\mathbf{B}_{N}^{R}$, mixes the two-electron singlet and triplet states, which allows for a leakage current through the DQD.

The leakage current $I(B)$ through an SWCNT DQD as function of an external magnetic field $B$ was measured by Churchill et al. [10]. Their data (Fig. 3f in [10]) show a zero-field peak with width of $\sim 6 \mathrm{mT}$. From the peak width the authors infer the HFC strength as $A=1-2 \times 10^{-4} \mathrm{eV}$, using the JN theory that predicts that the peak width is proportional to the HFC if large detuning $(\Delta \gg t)$, large nuclear fields $\left(B_{N} \gg \Delta_{\mathrm{ST}}\right)$, and the dominance of inelastic (e.g., phonon-mediated) interdot tunneling are assumed.

We wish to draw attention to a potential alternative interpretation of the zero-field peak of $I(B)$ found in [10], which does not invoke the assumption of large nuclear fields. By analyzing the JN model in the regime of large detuning $\Delta \gg t$, but small nuclear fields $B_{N} \ll \Delta_{\mathrm{ST}}$ and no inelastic interdot tunneling, we find that the functional form of $I(B)$ is approximately a Lorentzian,

$$
I(B) \sim \frac{B_{N}^{2}}{t^{2}} \frac{\gamma^{2}}{\gamma^{2}+B^{2}} e \Gamma_{R},
$$

where $e$ is the electron charge, and $\Gamma_{R}$ is the tunneling rate of electrons from the on-site singlet state to the right lead. Most importantly, the width of the Lorentzian in Eq. (8) is obtained as $\gamma=\sqrt{3 / 8} \Delta_{\mathrm{ST}}$, i.e., it is independent of the HFC in contrast to the original analysis in Ref. [10]. Derivation and discussion of Eq. (8) is provided in [29].

We finally note that theoretical efforts focused on understanding the anomalously large HFC in the transport measurement and found the possibility of an increased HFC in TLL systems but limited to the millikelvin temperature range $[40,41]$.

In conclusion, we developed the theory of NMR spinlattice relaxation time in a Tomonaga-Luttinger liquid which enabled to determine the electron-nucleus hyperfine coupling constant in carbon nanotubes. The value is in disagreement with that deduced from transport measurements in quantum dots made of ${ }^{13} \mathrm{C}$ enriched SWCNTs. We have reanalyzed the latter experiment in a different regime of the JN theory, which cures this discrepancy.

Work supported by the Hungarian State Grants (OTKA) Nr. K72613 and CNK80991, by the Austrian Science Funds (FWF) project Nr. P21333-N20, by the European Research Council Grant Nr. ERC-259374-Sylo, by the Marie Curie Grants PIRG-GA-2010-276834 and PERG08-GA-2010-276805, and by the New Széchenyi Plan Nr. TÁMOP-4.2.1/B-09/1/KMR-2010-0002. B. D. acknowledges the Bolyai programme of the Hungarian Academy of Sciences. A. K. acknowledges the Magyary programme and the EGT Norway Grants.

*ferenc.simon@univie.ac.at

[1] B. Kane, Nature (London) 393, 133 (1998).

[2] C. P. Slichter, Principles of Magnetic Resonance (SpingerVerlag, New York, 1989), 3rd ed.

[3] I. Žutić, J. Fabian, and S. D. Sarma, Rev. Mod. Phys. 76, 323 (2004).

[4] A. Abragam, Principles of Nuclear Magnetism (Oxford University Press, Oxford, England, 1961).

[5] S. A. Perera, J. D. Watts, and R. J. Bartlett, J. Chem. Phys. 100, 1425 (1994).

[6] D. J. E. Ingram, Free Radicals as Studied by Electron Spin Resonance (Butterworths Scientific Publications, London, 1958).

[7] C. H. Pennington and V. A. Stenger, Rev. Mod. Phys. 68, 855 (1996).

[8] O. V. Yazyev, Nano Lett. 8, 1011 (2008).

[9] H. J. Mcmanus, R. W. Fessenden, and D. M. Chipman, J. Phys. Chem. 92, 3781 (1988).

[10] H. O. H. Churchill, A. J. Bestwick, J. W. Harlow, F. Kuemmeth, D. Marcos, C.H. Stwertka, S. K. Watson, and C. M. Marcus, Nature Phys. 5, 321 (2009). 
[11] O. N Jouravlev and Y. Nazarov, Phys. Rev. Lett. 96, 176804 (2006).

[12] C. G. Van de Walle and P. E. Blöchl, Phys. Rev. B 47, 4244 (1993).

[13] A. Pályi and G. Burkard, Phys. Rev. B 80, 201404(R) (2009).

[14] X.-P. Tang, A. Kleinhammes, H. Shimoda, L. Fleming, K. Y. Bennoune, S. Sinha, C. Bower, O. Zhou, and Y. Wu, Science 288, 492 (2000).

[15] C. GozeBac, S. Latil, L. Vaccarini, P. Bernier, P. Gaveau, S. Tahir, V. Micholet, R. Aznar, A. Rubio, K. Metenier, and F. Beguin, Phys. Rev. B 63, 100302(R (2001).

[16] P. M. Singer, P. Wzietek, H. Alloul, F. Simon, and H. Kuzmany, Phys. Rev. Lett. 95, 236403 (2005).

[17] Y. Ihara, P. Wzietek, H. Alloul, M. H. Rümmeli, T. Pichler, and F. Simon, Europhys. Lett. 90, 17004 (2010).

[18] M. Bockrath, D. H. Cobden, J. Lu, A. G. Rinzler, R.E. Smalley, L. Balents, and P. L. McEuen, Nature (London) 397, 598 (1999).

[19] Z. Yao, H.W. C. Postma, L. Balents, and C. Dekker, Nature (London) 402, 273 (1999).

[20] H. Ishii et al., Nature (London) 426, 540 (2003).

[21] H. Rauf, T. Pichler, M. Knupfer, J. Fink, and H. Kataura, Phys. Rev. Lett. 93, 096805 (2004).

[22] S. Tomonaga, Prog. Theor. Phys. 5, 349 (1950).

[23] J. M. Luttinger, J. Math. Phys. (N.Y.) 4, 1154 (1963).

[24] B. Dóra, M. Gulácsi, F. Simon, and H. Kuzmany, Phys. Rev. Lett. 99, 166402 (2007).

[25] B. Gao, A. Komnik, R. Egger, D. C. Glattli, and A. Bachtold, Phys. Rev. Lett. 92, 216804 (2004).

[26] A factor 4 appears in Eq. (2) if the DOS is defined as states $/(\mathrm{eV} \cdot$ atom $)$.
[27] We note that Ref. [14] cites a value from Ref. [28] that is a factor 2 larger due to neglect of the spin degeneracy.

[28] J. W Mintmire and C. T White, Phys. Rev. Lett. 81, 2506 (1998).

[29] See Supplemental Material at http://link.aps.org/ supplemental/10.1103/PhysRevLett.107.187204 for details.

[30] J. Voit, Rep. Prog. Phys. 58, 977 (1995).

[31] F. Simon, C. Kramberger, R. Pfeiffer, H. Kuzmany, V. Zólyomi, J. Kürti, P. M. Singer, and H. Alloul, Phys. Rev. Lett. 95, 017401 (2005).

[32] O. V. Yazyev and L. Helm, Phys. Rev. B 72, 245416 (2005).

[33] J. Fischer and B. Trauzettel, and D. Loss, Phys. Rev. B 80, 155401 (2009).

[34] L. Mayrhofer and M. Grifoni, Eur. Phys. J. B 63, 43 (2008).

[35] K. Ono, D. G. Austing, Y. Tokura, and S. Tarucha, Science 297, 1313 (2002).

[36] F. H. L. Koppens, J. A. Folk, J. M. Elzerman, R. Hanson, L.H.W. van Beveren, I. T. Vink, H.P. Tranitz, W. Wegscheider, L.P. Kouwenhoven, and L.M. K. Vandersypen, Science 309, 1346 (2005).

[37] I. A. Merkulov, A. L. Efros, and M. Rosen, Phys. Rev. B 65, 205309 (2002).

[38] S. I. Erlingsson and Y. V. Nazarov, Phys. Rev. B 66, 155327 (2002).

[39] This is not to be mistaken with the singlet-triplet gap for a doubly occupied QD.

[40] B. Braunecker, P. Simon, and D. Loss, Phys. Rev. B 80, 165119 (2009).

[41] B. Braunecker, P. Simon, and D. Loss, Phys. Rev. Lett. 102, 116403 (2009). 